ARTIGO ORIGINAL ORIGINAL ARTICLE

\section{Impacto econômico da interrupção do tratamento com inibidor de tirosina quinase em pacientes com leucemia mieloide crônica em um hospital federal referência em oncologia}

\author{
Economic impact of discontinuing treatment with \\ tyrosine kinase inhibitor in patients with chronic myeloid \\ leukemia at a federal hospital reference in oncology \\ Daniel de Paiva D'Avila', Dulce Helena Nunes Couto², Rodrigo Saar da Costa²
}

DOI: $10.21115 / J B E S . v 13 . n 2 . p 108-16$

\section{Palavras-chave:}

economia da saúde, leucemia mielogênica crônica BCR-ABL positiva, proteínas tirosina quinase

\section{Keywords:}

health economics, BCR-ABL positive chronic myelogenous leukemia, protein tyrosine kinases

\section{RESUMO}

Objetivo: Avaliar o impacto econômico da descontinuação do tratamento da leucemia mieloide crônica (LMC) com inibidores da tirosina quinase (ITQs) em primeira ou segunda linha. Métodos: O modelo incluiu pacientes com diagnóstico de LMC em tratamento com ITQs que iniciaram o tratamento até 2012, em condições elegíveis no ano de 2015. Foi considerado um horizonte temporal de cinco anos sob a perspectiva do sistema público de saúde. Custos associados ao tratamento, como medicamento, monitoramento e manejo de eventos adversos, foram analisados. A avaliação foi composta por dois cenários: o cenário referência, com uso contínuo do medicamento, e o cenário comparador, com a interrupção do tratamento medicamentoso. Ambos os cenários consideraram as tecnologias disponíveis no período de 2015 a 2019. A análise de sensibilidade propôs variações nos cenários com a finalidade de avaliar a robustez do modelo. Além disso, uma extrapolação para nível nacional foi realizada, utilizando dados epidemiológicos para a obtenção do número de pacientes. Resultados: Foram selecionados 268 pacientes que iniciaram o tratamento até 2012. Desses, 65 foram elegíveis à descontinuação. A análise econômica mostrou uma economia de $\mathrm{R} \$ 670.558,10$ no primeiro ano, uma economia acumulada em cinco anos de $\mathrm{R} \$ 3.665 .355,98$ e de $\mathrm{R} \$$ 66.517.232,80 no contexto institucional e nacional, respectivamente. A análise de sensibilidade foi favorável em todos os cenários propostos. Conclusões: A descontinuidade do tratamento da LMC mostrou-se, economicamente, uma importante oportunidade sob a perspectiva do sistema de saúde em flexibilizar novos investimentos tecnológicos e/ou ampliação de acesso, além da melhoria na qualidade de vida do paciente.

\footnotetext{
ABSTRACT

Objective: To assess the economic impact of discontinuing treatment of chronic myeloid leukemia (CML) with first or second line tyrosine kinase inhibitors (ITQs). Methods: The model included patients diagnosed with CML undergoing treatment with ITQs who started treatment until 2012, under eligible conditions in the year 2015. A 5-year time horizon was considered from the perspective of the public health system. Costs associated with treatment, such as medication, monitoring and handling adverse events were analyzed. The evaluation consisted of two scenarios, the reference scenario with continuous use of the drug and the comparator scenario with the interruption of drug treatment. Both scenarios considered the technologies available in the period from 2015 to 2019. The sensitivity

Recebido em: 01/06/2021. Aprovado para publicação em: 12/07/2021.

1. Farmacêutico-residente no Serviço de Farmácia do Hospital do Câncer I, Instituto Nacional de Câncer José Alencar Gomes da Silva, Rio de Janeiro, RJ, Brasil.

2. Tecnologista farmacêutico no Serviço de Farmácia do Hospital do Câncer I, Instituto Nacional de Câncer José Alencar Gomes da Silva, Rio de Janeiro, RJ, Brasil.

Financiamento: Não houve nenhuma fonte de financiamento.

Instituição onde o trabalho foi executado: Instituto Nacional de Câncer José Alencar Gomes da Silva (Inca).

Congressos: Este trabalho não foi apresentado em nenhum congresso.

Autor correspondente: Rodrigo Saar da Costa. Hospital do Câncer I - Instituto Nacional de Câncer José Alencar Gomes da Silva. Praça Cruz Vermelha, 23, 70 andar, Centro, Rio de Janeiro, RJ, Brasil. CEP: 20230-130. Telefone: +55 (21) 99654-8981. E-mail: saarrc@gmail.com
} 
analysis proposed variations in the scenarios in order to assess the robustness of the model. In addition, an extrapolation to the national level was performed, using epidemiological data to obtain the number of patients. Results: 268 patients who started treatment until 2012 were selected. Of these, 65 were eligible for discontinuation. The economic analysis showed savings of $\mathrm{R} \$ 670,558.10$ in the first year, accumulated savings in five years of $R \$ 3,665,355.98$ and $R \$ 66,517,232.80$ in the institutional and national context, respectively. The sensitivity analysis was favorable in all the proposed scenarios. Conclusions: The discontinuity of CML treatment proved to be, economically, an important opportunity from the perspective of the health system in making new technological investments and / or expanding access more flexible, in addition to improving the patient's quality of life.

\section{Introdução}

A leucemia mieloide crônica (LMC) está entre as mais prevalentes entre os diversos tipos de leucemia e representa cerca de $15 \%$ delas. A estimativa mundial é de 249 mil novos casos de leucemia, sendo o décimo tumor mais incidente entre todos os cânceres (American Cancer Society, 2020). No Brasil, a estimativa para cada ano do triênio 2020-2022 é de 5.920 casos em homens e 4.890 novos casos em mulheres, com um risco estimado de 5,67 e 4,56 casos novos para cada 100 mil homens e mulheres, respectivamente (Inca, 2020).

A LMC é uma doença modelo para terapia direcionada, caracterizada pela presença do cromossoma Philadelphia e seu equivalente molecular, o gene de fusão BCR-ABL (Druker et al., 2006). Esse marcador leucêmico específico que codifica uma proteína quimérica com atividade tirosina quinase elevada é considerado o condutor do processo leucêmico (Heisterkamp et al., 1983). A história natural da doença foi revolucionada a partir da década de 1990, em que novos fármacos foram desenvolvidos, entre eles o mesilato de imatinibe, capaz de ocupar o sítio ativo da tirosina quinase BCR-ABL e interromper o fenótipo maligno da doença (Hehlmann et al., 2007). Um estudo internacional, o IRIS, comprovou a superioridade do tratamento com o primeiro inibidor de tirosina quinase (ITQ) - imatinibe -, em relação ao tratamento-padrão com interferon (O'Brien et al., 2003).

Os resultados do tratamento e as taxas de sobrevida para pacientes com leucemia mieloide crônica em fase crônica (LMC-FC) melhoraram substancialmente desde a introdução do imatinibe e o subsequente desenvolvimento de ITQs de segunda geração (Kantarjian et al., 2012; Cortes et al., 2016). Com esse advento, a maioria dos pacientes com LMC-FC alcançou resposta molecular maior (RM), chegando a respostas moleculares profundas, como meta de tratamento (Etienne et al., 2016; Hochhaus et al., 2009).

Respostas moleculares profundas são definidas como: RM 4 (redução de 4 log da linha de base do IRIS) ou doença detectável $\leq$ 0,01\% BCR-ABL IS; RM 4,5 (redução de 4,5 log a partir da linha de base do IRIS) ou doença detectável $\leq$ 0,0032\% BCR-ABL IS; RM 5 (redução de 5 log a partir da linha de base do IRIS) ou doença detectável $\leq 0,001 \%$ BCR-ABL IS (Cross et al., 2015; Hughes et al., 2006). No entanto, os ITQs não são considerados curativos e são administrados diariamente de maneira contínua e indefinida (Kantarjian et al.,
2012; Huang et al., 2012). Como consequência, o tratamento e os cuidados de alta qualidade devem não apenas integrar o controle ideal da doença, mas também o gerenciamento de eventos adversos (EAs) a curto e longo prazo (Rea, 2015).

No Brasil, o imatinibe, é o principal ITQ utilizado na LMC-FC, visto que os ITQs de segunda geração, como o dasatinibe e o nilotinibe são utilizados como segunda linha de tratamento no Sistema Único de Saúde (SUS). Apesar de demonstrarem uma maior taxa de reposta precoce, não mostraram impacto na sobrevida global, sendo utilizados em caso de falha, resistência ou intolerância terapêutica ao imatinibe (Brasil, 2020; Fachi et al., 2018).

Considerando que os pacientes que respondem bem à terapia com ITQ tenham taxas de sobrevivência semelhantes às observadas na população geral, o impacto da terapia com ITQ a longo prazo na vida dos pacientes deve ser considerado (Huang et al., 2012). A dor osteoarticular foi o EA mais comum e significativamente mais frequente na coorte de imatinibe, seguida de manifestações dermatológicas, astenia e diarreia (Bettiol et al., 2018). Com os ITQs de segunda geração, os EAs clínicos mais comuns relatados para o nilotinibe foram rash, prurido, cefaleia, diarreia e fadiga, sendo a cardiotoxicidade o EA mais grave pelo prolongamento do intervalo QT (Bauer et al., 2016). O dasatinibe mostrou EAs semelhantes, sendo os mais comuns cefaleia, dor musculoesquelética, diarreia, infecção, fadiga e rash, e toxicidade pulmonar como EA mais grave, pela ocorrência de derrame pleural e hipertensão arterial pulmonar (Breccia et al., 2016).

Os pacientes que alcançam respostas moleculares profundas apresentam baixo risco de progressão da doença para fases mais agressivas, fase acelerada e crise blástica, alta taxa de sobrevivência a longo prazo e a possibilidade de descontinuação do tratamento com ITQ (Hehlmann et al., 2014). Ensaios clínicos de descontinuação demonstraram que, em pacientes com respostas moleculares profundas sustentadas e critérios bem-definidos em guidelines internacionais, o tratamento com ITQ pode ser suspenso com segurança, com manutenção da resposta molecular (Etienne et al., 2016; Saußele et al., 2016). Em aproximadamente 50\% dos pacientes que descontinuaram a terapia com ITQ, foi mantida a remissão da doença sem tratamento (Hehlmann et al., 2019; Saussele et al., 2018). Dentre eles, podem-se destacar dois grandes estudos europeus. O projeto STIM, realizado na França, selecionou pacientes elegíveis, em que aproximada- 
mente $40 \%$ dos pacientes que interromperam o tratamento mantiveram a remissão da doença sem tratamento, e os demais (60\%) tiveram recidiva da doença, porém, ao retomarem o uso do medicamento, restabeleceram os níveis indetectáveis do transcrito BCR-ABL (Etienne et al., 2016). O EURO-SKI, realizado em 61 centros de investigação distribuídos em 11 países europeus, obteve resultados semelhantes, com aproximadamente $60 \%$ dos pacientes com remissão sem tratamento (Saussele et al., 2018).

Essa nova abordagem terapêutica traz benefícios tanto para o paciente, evitando os EAs comuns ao uso dos ITQs e melhorando a qualidade de vida, desde que atingidos os critérios estabelecidos internacionalmente e com o monitoramento imprescindível por meio de reação em cadeia da polimerase transcriptase reversa quantitativa em tempo real (RT-PCR), quanto para a gestão em saúde no Brasil, pela redução de custos do tratamento da LMC.

A avaliação econômica é uma ferramenta fundamental no desenvolvimento dos sistemas de saúde, capaz de auxiliar instituições no processo de tomada de decisões, lidando com a integração entre ciência e política de saúde. Dessa forma, avaliar, sob o ponto de vista econômico, o impacto da referida proposta terapêutica torna-se preponderante e de extrema relevância para o cenário no SUS (Costa et al., 2017). O objetivo deste estudo foi avaliar o impacto econômico da descontinuação do tratamento da LMC com ITQs em primeira ou segunda linha em um hospital federal referência em oncologia.

\section{Métodos}

A análise de impacto orçamentário (AIO) é definida como uma avaliação das consequências financeiras decorrentes da incorporação ou remoção de uma dada tecnologia em saúde em um cenário específico e de recursos finitos. Trata-se de uma importante ferramenta amplamente utilizada na tomada de decisão do gestor público (Ferreira-Da-Silva et al., 2012).

O estudo compreende uma análise de uma coorte retrospectiva de um hospital federal referência em oncologia, sob a perspectiva do gestor público. Foram incluídos no estudo pacientes com diagnóstico de LMC-FC, com idade mínima de 18 anos e sob o tratamento com ITQ de primeira ou segunda linha pelo tempo mínimo de três anos, apresentando resposta molecular profunda (RM 4 ou RM 4,5) por pelo menos dois anos e, no mínimo, seis exames de RT-PCR em dois anos seguidos, no ano de 2015.

Dados clínicos como idade, sexo, fase da doença, reações adversas durante o tratamento, exames laboratoriais, medicamento ITQ atual, tratamento prévio, início do tratamento com ITQ e seu tempo de uso foram obtidos por meio de prontuários físico e eletrônico da instituição.

Na proposta do contexto nacional, foi utilizada a estimativa do Instituto Nacional de Câncer José Alencar Gomes da Silva (Inca) para câncer no triênio 2020-2022 para seleção da amostra. Nesse contexto, foi considerado que 25\% da população estimada atendia às condições de elegibilidade citadas anteriormente de acordo com a proporção encontrada no contexto institucional.

Foi utilizado um horizonte temporal de cinco anos de acordo com as recomendações das diretrizes metodológicas para AIO do sistema de saúde. Segundo a tábua de mortalidade do Instituto Brasileiro de Geografia e Estatística (IBGE) 2020, a expectativa de vida da população quando atinge 45 anos é de 80,2 anos. Portanto, foi utilizado um horizonte temporal de 35 anos para análise dos dados em nível nacional.

Os custos diretos referentes aos procedimentos (consultas, exames laboratoriais, internação e visitas ao serviço de emergência) foram obtidos a partir de valores de reembolso do SUS registrados no sítio do Sistema de Gerenciamento da Tabela de Procedimentos, Medicamentos, Órteses, Próteses e Materiais e Medicamentos Especiais do SUS (SIGTAP-SUS). O preço dos ITQs imatinibe, dasatinibe e nilotinibe e os medicamentos de suporte para manejo de EAs foram consultados na base de dados do sistema interno da instituição que realiza as aquisições por meio de licitações públicas, referente ao ano de 2019.

Para calcular o custo relacionado ao uso do ITQ, foi utilizada, como dose diária, a recomendação da Conitec sobre Protocolo Clínico e Diretrizes Terapêuticas da Leucemia Mieloide Crônica do Adulto. Devido ao baixo número de pacientes em uso de ITQs de segunda linha, foi utilizado a média dos preços do dasatinibe e nilotinibe como custo dos ITQs de segunda linha. O custo do exame RT-PCR foi retirado do relatório da Conitec para o diagnóstico e monitoramento da LMC (Brasil, 2019).

Como ferramenta para cálculo de impacto orçamentário, foi utilizada uma adaptação da Tabela Brasileira de Impacto Orçamentário de Tecnologias da Saúde do Ministério da Saúde, desenvolvida para fármacos, disponível para download no sítio da Rede Brasileira de Tecnologias em Saúde, e o Manual de Impacto Orçamentário (Brasil, 2014b).

A avaliação de impacto orçamentário baseou-se em dois cenários: um de referência e outro comparador. O cenário referência foi composto pelo tratamento atual, ou seja, uso contínuo do medicamento ITQ. O cenário comparador foi composto pela nova opção de tratamento, ou seja, com a interrupção do tratamento medicamentoso. De acordo com ensaios clínicos de descontinuação, cerca de 50\% dos pacientes, em média, podem ser beneficiados com a interrupção do ITQ, mantendo a remissão sem tratamento (Etienne et al., 2016; Saussele et al., 2018). O cenário referência considerou as tecnologias disponíveis durante o período de 2015 a 2019 para o tratamento de LMC e as respectivas proporções de consumo do uso de ITQ de primeira ou segunda linha de tratamento, segundo a análise de prontuários da instituição.

Para elaboração dos cenários supracitados, o cenário referência é representado pelo tratamento atual, em que os in- 
divíduos fazem uso regular e ininterrupto do medicamento, seja de primeira ou segunda linha. Para esse caso, foi considerado o custo relacionado ao uso regular do medicamento, o custo relacionado à realização do exame de RT-PCR, para monitoramento, a cada três meses, além do custo relacionado ao manejo dos possíveis EAs decorrentes do uso contínuo dos ITQs.

No cenário comparador, no qual se propõe a interrupção do tratamento, foram considerados dois grupos. No grupo remissão, 50\% dos pacientes mantêm a remissão da doença sem tratamento, e no grupo recidiva, considera-se que $50 \%$ apresentem recidiva da doença. No grupo remissão, foi considerado apenas o custo relacionado à realização do exame de RT-PCR para monitoramento, porém esse exame passa a ser realizado a cada mês no primeiro ano, a cada dois meses no segundo ano e a cada três meses a partir do terceiro ano. Para o grupo recidiva, foram considerados o custo da reintrodução do uso de ITQ regularmente, o custo do exame de RT-PCR conforme o grupo remissão e o custo relacionado ao manejo dos possíveis EAs devido à retomada do uso do medicamento, como é representado na figura 1.

Para avaliar a robustez do modelo, foi realizada uma análise de sensibilidade univariada determinística. Foram propostos quatro cenários com o objetivo de avaliar variações na proporção de recidivas, custos dos medicamentos, proporção do uso dos ITQs de primeira ou segunda linha de tratamento e variações no número de pacientes elegíveis. No cenário 1, foi aplicada uma variação de $60 \%$ e $40 \%$, respectivamente, na taxa de recidiva da doença, considerando os extremos obtidos nos estudos de descontinuação (Etienne et al., 2016; Saussele et al., 2018). Para o cenário 2, foi utilizada uma redução em 40\% no preço dos ITQs de segunda linha devido à futura entrada dos genéricos desses medicamentos no mercado brasileiro após a queda da patente dos medicamentos de referência utilizados atualmente. Para o cenário 3, foi aplicado um aumento de 30\% na utilização dos ITQs de

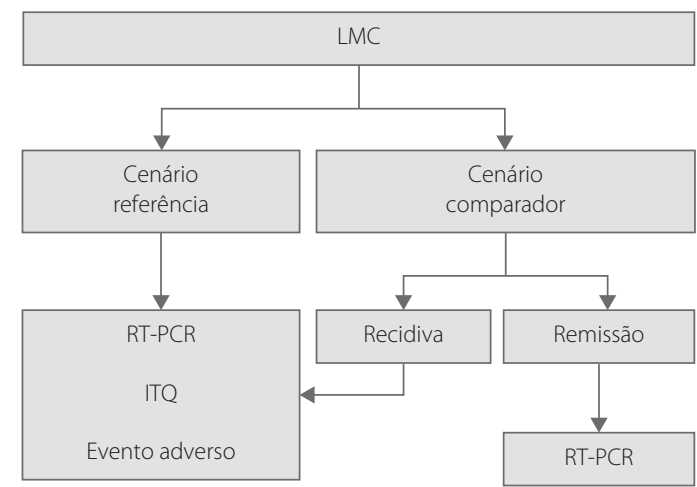

LMC: leucemia mieloide crônica; RT-PCR: reação em cadeia da polimerase transcriptase reversa; ITQ: inibidor da tirosina quinase.

Fonte: Elaboração própria.

Figura 1. Representação esquemática da composição dos cenários de referência e comparador. segunda linha, seguindo a proporção encontrada em outros estudos publicados sobre o assunto (Goulart, 2016). Por fim, o cenário 4 representa um aumento dos pacientes elegíveis, considerando o acréscimo de 45 pacientes que foram excluídos, sendo 35 por dados incompletos e 10 por falta do exame RT-PCR. A análise estatística foi realizada com o auxílio de uma planilha em Excel’ 2013, em que foi feita a média de frequência para os EAs avaliados.

Este projeto foi submetido à Plataforma Brasil e aprovado pelo Comitê de Ética do Inca sob o número de identificação CAAE: 39612720.1.0000.5274.

\section{Resultados}

Foram selecionados 268 pacientes que iniciaram o tratamento com ITQ até dezembro de 2012. Desses, foram excluídos 100 pacientes: 65 por óbito e 35 por possuírem dados incompletos. Dos 168 restantes, 103 não atenderam aos critérios de inclusão, sendo 88 excluídos devido à falta de resposta molecular profunda em dois anos ininterruptos, 10 por não possuírem pelo menos seis RT-PCR em dois anos seguidos, dois por estarem em uso de interferon e três por terem idade inferior a 18 anos, resultando em 65 pacientes elegíveis para a nova opção de tratamento a partir do ano de 2015.

A faixa etária dos indivíduos selecionados foi de 18 e 72 anos, com média e mediana de 45 e 47 anos, respectivamente, sendo 35 pertencentes ao sexo masculino e 30, ao sexo feminino. Quanto ao uso do ITQ, 58 indivíduos eram tratados com imatinibe, seis com dasatinibe e apenas um com nilotinibe.

Os EAs foram obtidos por meio do registro em prontuário físico no período de 2015 a 2019, e os mais frequentes foram dores musculoesqueléticas, encontradas em 34\% dos pacientes durante os cinco anos, febre (31\%), diarreia (25\%), tosse (25\%), náuseas e vômitos (22\%), cefaleia e enxaqueca (14\%), fadiga (14\%), neuropatia e parestesia (9\%), lesões de pele (9\%) e edemas (8\%). O custo de cada EA foi feito com base na tabela do SIGTAP, acessada em 2020, e no preço médio do sistema interno da instituição dos medicamentos envolvidos no manejo do EA. A tabela 1 mostra o custo dos medicamentos, exame RT-PCR e o custo do manejo dos EAs.

No cenário referência, estima-se um custo anual de R\$1.549.687,60 para os 65 indivíduos e um custo acumulado de $\mathrm{R} \$ 7.748 .437,99$ em cinco anos. No cenário comparador, estima-se um custo, no primeiro ano, de R\$ 879.129,50, no segundo ano, de R\$ 816.616,40 e, a partir do terceiro ano, um custo anual de R\$795.778,70, com um custo acumulado em cinco anos de $R \$ 4.083 .082,01$. Portanto, a opção por interrupção do tratamento pode proporcionar, no contexto institucional, uma economia anual média de $\mathrm{R} \$ 733.071,40$, com uma economia em cinco anos de R\$3.665.355,98 (Figura 2A).

Segundo estimativas do Inca, espera-se, para o triênio 2020-2022, cerca de 10.810 novos casos de leucemia a cada ano, sendo 5.920 para homens e 4.890 para mulheres. A LMC representa cerca de $15 \%$ de todas as leucemias, portanto es- 
tima-se cerca de 1.622 novos casos de LMC no país a cada ano do triênio, sendo 888 para homens e 734 para mulheres. Os dados foram extrapolados para um contexto nacional utilizando $25 \%$ da incidência nacional como critério de elegibilidade, resultando em uma economia anual média de R\$ 13.303.446,56, com uma economia acumulada em cinco anos de $\mathrm{R} \$$ 66.517.232,80 (Figura 2B e Figura 3).

Nesse estudo, a média de idade dos pacientes foi de 45 anos. Segundo o IBGE, a expectativa de vida da população brasileira aos 45 anos é de 80,2 anos. Portanto, foi calculada a incorporação da incidência a cada ano, no contexto nacional, obtendo-se um custo de R\$ 6.013.373.035,43 no cená- rio referência e um custo de $\mathrm{R} \$ 3.111 .233 .269,44$ no cenário comparador, resultando em uma economia nacional de R\$ 2.902.139.765,99 em 35 anos.

A análise de sensibilidade (Tabela 2) se mostrou favorável à interrupção em todos os cenários propostos, sendo o preço dos medicamentos fator principal, pelas variações encontradas nessa análise. A variação de $60 \%$ da taxa de recidiva do cenário 1 mostrou a menor economia no primeiro ano (R\$ 519.854,08), devido ao maior número de pacientes retomando o uso do ITQ. Por outro lado, na variação de 40\%, já era esperada uma economia superior à do primeiro, uma vez que possui a menor taxa de recidiva. Para o cenário 2, representado

Tabela 1. Custo dos ITQs, exame RT-PCR e manejo dos eventos adversos no ano de 2019

\begin{tabular}{|c|c|c|c|}
\hline Medicamento 1,2 & Valor unitário médio & Posologia & Custo anual \\
\hline Imatinibe 400 mg & $\mathrm{R} \$ 51,43$ & 400 mg/dia & $\mathrm{R} \$ 18.771,95$ \\
\hline Dasatinibe 100 mg & $\mathrm{R} \$ 137,36$ & 100 mg/dia & $R \$ 50.136,40$ \\
\hline Nilotinibe 200 mg & $R \$ 45,23$ & 800 mg/dia & $R \$ 66.035,80$ \\
\hline Custo médio 2a linha & - & - & $\mathrm{R} \$ 58.921,65$ \\
\hline \multirow[t]{2}{*}{ Exame RT-PCR 2,3} & Valor unitário médio & Exames/ano & Custo anual \\
\hline & $R \$ 160,29$ & 4 & $\mathrm{R} \$ 641,14$ \\
\hline \multirow{2}{*}{ Evento Adverso ${ }^{1,3}$} & \multirow{2}{*}{ Pacientes } & \multirow{2}{*}{ Episódios } & Custo $\mathrm{EA}^{*}$ \\
\hline & & & Custo EA total ${ }^{* *}$ \\
\hline \multirow{2}{*}{ Dor musculoesquelética } & \multirow{2}{*}{$22(34 \%)$} & \multirow{2}{*}{91} & $\mathrm{R} \$ 243,04$ \\
\hline & & & $R \$ 22.116,64$ \\
\hline \multirow{2}{*}{ Febre } & \multirow{2}{*}{$20(31 \%)$} & \multirow{2}{*}{40} & $R \$ 221,09$ \\
\hline & & & $R \$ 8.843,60$ \\
\hline \multirow{2}{*}{ Diarreia } & \multirow{2}{*}{$16(25 \%)$} & \multirow{2}{*}{34} & $\mathrm{R} \$ 344,77$ \\
\hline & & & $R \$ 11.722,18$ \\
\hline \multirow{2}{*}{ Tosse } & \multirow{2}{*}{$16(25 \%)$} & \multirow{2}{*}{26} & $\mathrm{R} \$ 13,76$ \\
\hline & & & $\mathrm{R} \$ 357,76$ \\
\hline \multirow{2}{*}{ Náuseas e vômitos } & \multirow{2}{*}{$14(22 \%)$} & \multirow{2}{*}{26} & $\mathrm{R} \$ 158,38$ \\
\hline & & & $\mathrm{R} \$ 4.117,88$ \\
\hline \multirow{2}{*}{ Cefaleia/Enxaqueca } & \multirow{2}{*}{$9(14 \%)$} & \multirow{2}{*}{14} & $\mathrm{R} \$ 231,81$ \\
\hline & & & $R \$ 3.245,34$ \\
\hline \multirow{2}{*}{ Fadiga/Fraqueza } & \multirow{2}{*}{$9(14 \%)$} & \multirow{2}{*}{18} & $\mathrm{R} \$ 170,13$ \\
\hline & & & $\mathrm{R} \$ 3.062,34$ \\
\hline \multirow{2}{*}{ Lesões de pele } & \multirow{2}{*}{$6(9 \%)$} & \multirow{2}{*}{29} & $\mathrm{R} \$ 162,48$ \\
\hline & & & $R \$ 4.711,92$ \\
\hline \multirow{2}{*}{ Neuropatia/Parestesia } & \multirow{2}{*}{$6(9 \%)$} & \multirow{2}{*}{12} & $R \$ 215,47$ \\
\hline & & & $R \$ 2.585,64$ \\
\hline \multirow{2}{*}{ Edemas } & $5(004)$ & 11 & $\mathrm{R} \$ 219,97$ \\
\hline & $J(8 \%)$ & 11 & $R \$ 2.419,67$ \\
\hline Custo EA/paciente/ano & & & $\mathrm{R} \$ 194,41$ \\
\hline
\end{tabular}

EA: evento adverso; ITQ: inibidor da tirosina quinase; RT-PCR: reação em cadeia da polimerase transcriptase reversa.

* Custo individual do manejo do EA. ** Custo total dos episódios de cada EA.

${ }^{1}$ Banco de dados da instituição. ${ }^{2}$ Conitec. ${ }^{3}$ SIGTAP.

Fonte: Elaboração própria. 
por uma redução de $40 \%$ nos preços do dasatinibe e nilotinibe, ocorre uma queda de 18\% em relação ao cenário-padrão. O cenário 3 apresenta um aumento de 37\% na economia em relação ao padrão, devido ao maior uso dos ITQs de segunda linha. Por fim, o cenário 4 apresentou a maior economia proposta no primeiro ano (R\$ 1.118.322,21), uma vez que a população elegível aumentou cerca de 15\%, representando uma economia 67\% maior, em comparação com o cenário-padrão.

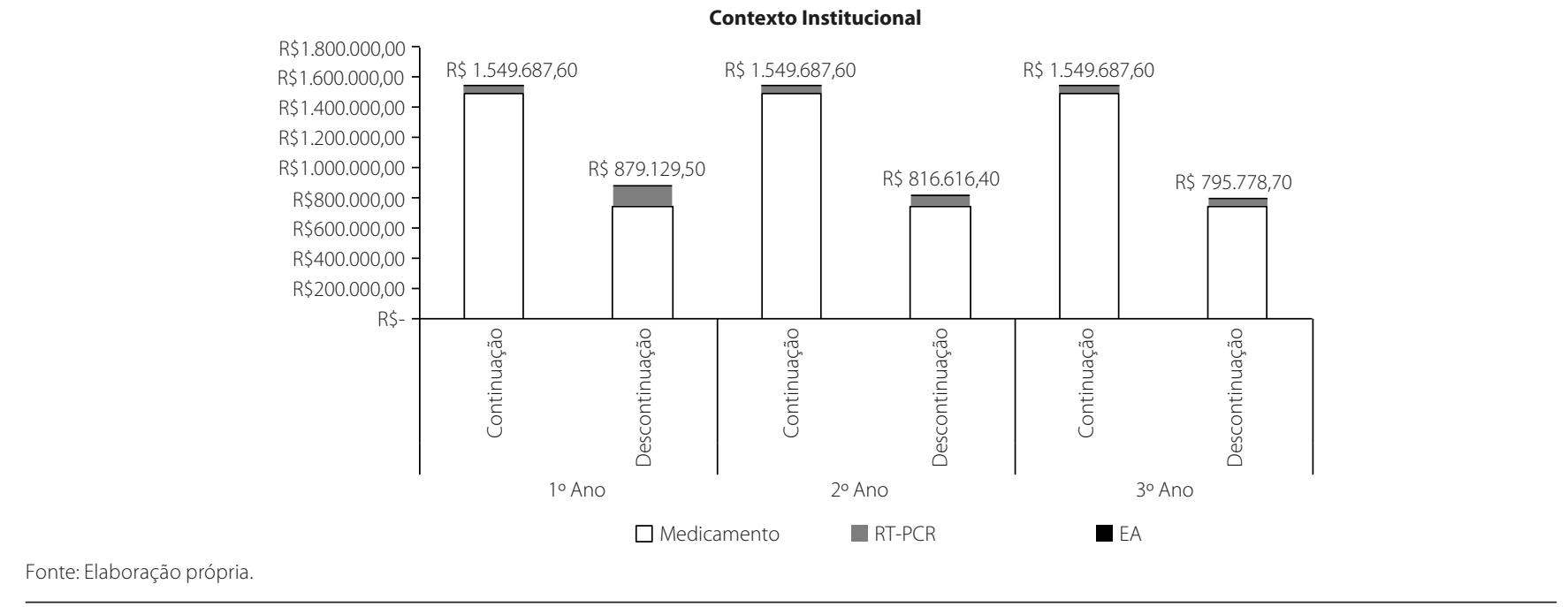

Figura 2A. Estimativa dos custos dos cenários referência e comparador no primeiro, segundo e terceiro ano no contexto institucional.

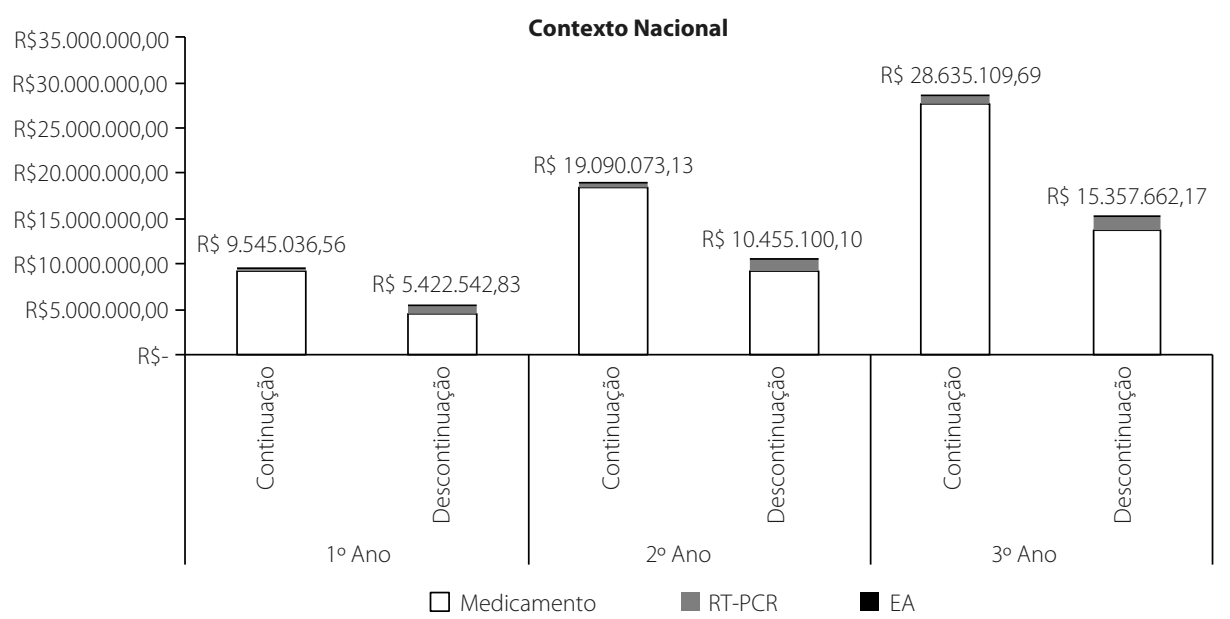

Fonte: Elaboração própria.

Figura 2B. Estimativa dos custos dos cenários referência e comparador no primeiro, segundo e terceiro ano no contexto nacional.

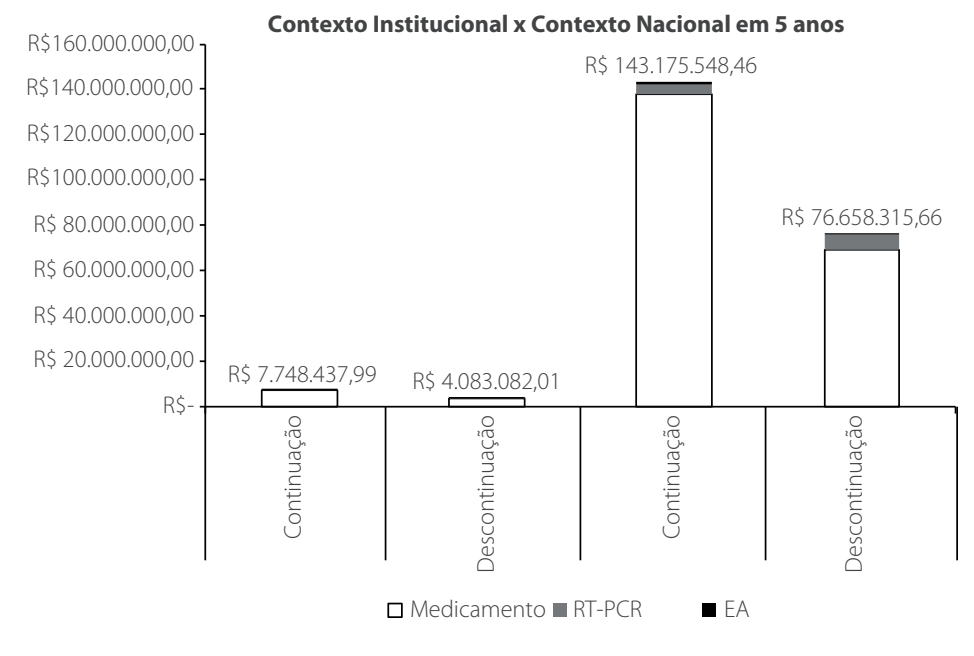

Fonte: Elaboração própria.

Figura 3. Estimativa de custo acumulado dos cenários referência e comparador no contexto institucional versus nacional em cinco anos. 
Tabela 2. Análise de sensibilidade univariada determinística do contexto institucional no primeiro ano

\begin{tabular}{|c|c|c|c|c|c|c|}
\hline \multirow[b]{2}{*}{ Cenário referência } & \multirow{2}{*}{$\begin{array}{c}\text { Cenário-padrão } \\
\text { (R\$) }\end{array}$} & \multicolumn{2}{|c|}{$\begin{array}{c}\text { Cenário } 1 \text { (R\$) } \\
60 \% 40 \% \\
\end{array}$} & \multirow{2}{*}{$\begin{array}{c}\text { Cenário } 2 \text { (R\$) } \\
1.305 .725,98\end{array}$} & \multirow{2}{*}{$\begin{array}{l}\text { Cenário } 3 \text { (R\$) } \\
2.060 .771,55 \\
\end{array}$} & \multirow{2}{*}{$\begin{array}{l}\text { Cenário } 4 \text { (R\$) } \\
2.589 .282,42\end{array}$} \\
\hline & & $1.549 .687,60$ & $1.549 .687,60$ & & & \\
\hline $\begin{array}{l}\text { Cenário } \\
\text { comparador }\end{array}$ & $879.129,50$ & $1.029 .833,52$ & $728.231,08$ & $757.148,69$ & $1.144 .057,45$ & $1.470 .960,21$ \\
\hline Economia & $670.558,10$ & $519.854,08$ & $821.456,52$ & $548.577,29$ & $916.714,10$ & $1.118 .322,21$ \\
\hline
\end{tabular}

Nota: * Cenário-padrão: referente ao contexto institucional. Cenário 1: variação da recidiva da doença em 60\% e 40\%, respectivamente. Cenário 2: redução de $40 \%$ no preço dos ITQs de segunda linha. Cenário 3: aumento da proporção do uso dos ITQs de segunda linha de tratamento para 30\%. Cenário 4: aumento da amostra elegível para $40 \%$

Fonte: elaboração própria.

\section{Discussão}

A remissão sem tratamento com a descontinuação do uso contínuo dos ITQs tornou-se uma nova perspectiva potencialmente promissora para a terapia da LMC, tornando-se cada vez mais uma nova opção viável de tratamento. Takahashi et al. (2012) analisaram 43 pacientes que descontinuaram o uso do imatinibe; desses, aproximadamente 56\% mantiveram resposta molecular sustentada; sem recorrência molecular após a descontinuação (Takahashi et al., 2012).

No estudo HOVON 51, trinta e três pacientes foram randomizados entre a continuação do imatinibe (braço A com 18 participantes) ou a descontinuação (braço $B$, com 15 participantes). A análise revelou 56\% e $61 \%$ de recidivas em um e dois anos desde a interrupção em pacientes que descontinuaram o imatinibe. Todos os pacientes avaliáveis permaneceram sensíveis ao imatinibe após a reinicialização e recuperaram a resposta molecular (Thielen et al., 2013).

Esses estudos mostram que a interrupção do uso do ITQ é um processo seguro, que pode beneficiar cerca de $50 \%$ dos indivíduos que adotarem essa nova abordagem terapêutica, desde que sejam avaliadas as condições clínicas e laboratoriais dos pacientes e que sejam respeitados os critérios de elegibilidade à descontinuação.

EAs a medicamentos são considerados uma questão de saúde pública, sendo o seu monitoramento e notificação compulsória de extrema importância para a segurança do paciente (Brasil, 2014a). Devido a sua frequência e gravidade, os EAs são considerados como um dos principais fatores relacionados à não adesão medicamentosa tanto em doenças crônicas quanto no tratamento oncológico (Menon, 2017). A resposta ao tratamento está relacionada diretamente ao uso correto do medicamento, sendo a baixa adesão um fator de risco para evolução da doença, com perda de resposta molecular (Marin et al., 2010; Noens et al., 2009).

O uso dos ITQs a longo prazo no tratamento da LMC é responsável por apresentar diversos EAs. Em um artigo de revisão publicado em 2016 por Breccia et al., o edema foi o EA mais comum, enquanto neste estudo ele foi o menos frequente. Porém, ambos os estudos encontraram a dor musculoesquelética como um dos EAs mais frequentes (Breccia et al., 2016). Outro estudo apresentando resultados semelhantes foi publicado por Caldemeyer et al. (Caldemeyer et al., 2016). Kizaki et al., em 2019, em um estudo sobre avaliação de eficácia e segurança do uso dos ITQs para tratamento de LMC, relataram como principais eventos o derrame pleural, dor no peito, derrame pericárdico, além dos EAs já descritos nos estudos anteriores, sendo esses eventos mais relacionados ao uso do dasatinibe e nilotinibe (Kizaki et al., 2019).

A ocorrência de EAs possui impacto na qualidade de vida dos pacientes, uma vez que é necessário o uso contínuo desses medicamentos. Assim, é comum entre os pacientes a interrupção do tratamento sem o consentimento médico, já que a ocorrência de EAs é um dos principais motivos para a não adesão ao tratamento (Almeida et al., 2014).

É importante ressaltar que o custo estimado para manejo dos EAs desse estudo possui baixo custo, cerca de R\$194,41 por paciente por ano, comparado ao custo diário dos ITQs. Porém, pode causar impacto significativo no dia a dia dos pacientes, e a ausência desses EAs proporcionar melhor qualidade de vida. As informações acerca dos EAs foram retiradas dos registros em prontuário físico, o que pode levar à subnotificação devido à falta de clareza nas informações encontradas, assim como à dificuldade de compreensão da escrita manual, caracterizando um potencial viés de informação.

A realização do exame molecular RT-PCR é fundamental para o monitoramento da resposta molecular do paciente durante o tratamento. Na proposta de descontinuação, é importante que seja garantida a realização desses exames regularmente, uma vez que a avaliação da resposta molecular profunda constitui o principal fator de elegibilidade, além do aumento da taxa de realização do exame durante os primeiros anos após a descontinuação do medicamento para avaliação da remissão ou recidiva da doença.

Yamazaki et al. (2020), em um estudo de impacto orçamentário da interrupção do uso de nilotinibe no Japão, avaliaram 901 pacientes em um horizonte temporal de três anos, com resultados de US\$ 66.567.775,00 (R\$ 267.136.481,08). O estudo considerou o custo dos medicamentos, o custo da visita médica e o custo do RT-PCR. O custo relacionado ao manejo de EAs foi omitido, pois foram considerados de baixo impacto (Yamazaki et al., 2020). Elias et al. (2019) analisa- 
ram, economicamente, a descontinuação do uso de ITQs de primeira ou segunda linha de 83 pacientes em horizonte temporal de quatro anos, com uma economia de 7,1 milhões ( $R \$ 28.492 .300,00$ ). $\mathrm{O}$ estudo considerou apenas o custo direto relacionado ao medicamento (Elias et al., 2019). Ambos os estudos divergem sobre os centros de custo considerados, porém, mesmo com as diferenças nas considerações em relação aos custos do manejo de EAs, exame de RT-PCR ou despesas médicas, observa-se que a maior parte do orçamento recai sobre os ITQs que possuem alto custo.

Quanto à análise econômica, observa-se uma economia significativa na proposta de descontinuação do medicamento. No cenário referência, estimam-se um custo anual de R\$ 1.549.687,60 e um custo acumulado de R\$ 7.748.437,99 em cinco anos. No cenário comparador, estima-se um custo, em cinco anos, de $R \$ 4.083 .082,01$. Dito isso, a opção por interrupção do tratamento pode proporcionar à instituição uma economia, em cinco anos, de $\mathrm{R} \$ 3.665 .355,98$, que representa aproximadamente $47 \%$ dos gastos com tratamento no cenário referência.

Shih et al. (2019), em uma análise comparativa sobre o uso de ITQs de segunda geração em relação ao imatinibe, estimaram um custo médio do tratamento com imatinibe genérico em \$35.000/ano nos EUA, em conversão direta seria equivalente a $R \$ 135.450,00$. Para os ITQs de segunda geração, utilizaram um custo de \$152.814/ano para o nilotinibe (R\$591.390,18) e \$232.417/ano para o dasatinibe (R\$ 899.453,79) (Shih et al., 2019).

Um dos principais fatores que impactaram essa análise está relacionado ao preço dos medicamentos praticados nesses países, que possuem influência direta no sistema de financiamento da saúde. Diferente dos EUA, o Brasil possui um sistema público de saúde. Os resultados evidenciaram um custo significativamente menor no Brasil, uma vez que foi utilizado o preço médio de aquisição em licitações, em que os valores de unidade do medicamento possuem um preço expressivamente menor que o preço praticado no mercado, tendo como uma de suas vantagens a negociação em grandes quantidades, por meio da compra centralizada pelo Ministério da Saúde.

$\mathrm{Na}$ análise de sensibilidade, foi demonstrada uma economia importante em todos os cenários propostos. E, mesmo em meio a incertezas, a interrupção de tratamento ainda permanece uma opção economicamente viável para o setor público.

A interrupção do tratamento traz benefícios tanto para o paciente, por evitar potenciais EAs decorrentes do uso dos ITQs, quanto para a gestão pública, devido à redução nos gastos constantes e cumulativos com o tratamento da doença, em que os números de novos casos permanecem crescentes, desde que garantida as condições de monitoramento, por meio de exames periódicos, como neste caso o RT-PCR, de forma que traga confiança para os pacientes que consintam em descontinuar o tratamento conforme critério clínicos preestabelecidos.

\section{Conclusão}

No Brasil, a descontinuação dos ITQs no tratamento da LMC é objeto de estudos clínicos e não é uma realidade na prática clínica no SUS. A análise econômica apresentada neste estudo representa uma projeção do impacto econômico considerando as características clínicas e laboratoriais dos pacientes elegíveis à interrupção do medicamento, conforme guidelines internacionais.

Os resultados mostraram que essa nova abordagem terapêutica pode proporcionar à instituição uma economia anual média de R\$733.071,20. Quando extrapolado para um cenário nacional, a economia anual média projetada foi de R\$ 13.303.446,56. Em um cenário de recursos finitos, em que a demanda por novas tecnologias e entrada de pacientes é sempre maior que os recursos disponíveis para o desenvolvimento desses serviços, deve-se considerar a economia gerada pela descontinuação do tratamento.

A descontinuidade do tratamento da LMC mostrou-se, economicamente, uma importante oportunidade sob a perspectiva do sistema de saúde pública em flexibilizar novos investimentos tecnológicos e ampliação de acesso. Além do ganho econômico, deve-se considerar a melhoria da qualidade de vida dos pacientes devido à ausência dos EAs relacionados ao medicamento, além da possibilidade de gestação em mulheres jovens.

\section{Referências bibliográficas}

Almeida MH de, Fogliatto L, Couto D. Importance of adherence to BCR-ABL tyrosine-kinase inhibitors in the treatment of chronic myeloid leukemia. Rev Bras Hematol Hemoter. 2014;36(1):54-9.

American Cancer Society. Key Statistics for Chronic Myeloid Leukemia. Available from: https://www.cancer.org/cancer/chronic-myeloidleukemia/about/statistics.html. Accessed on: Sep 21, 2020

Bauer S, Buchanan S, Ryan I. Tyrosine Kinase Inhibitors for the Treatment of Chronic-Phase Chronic Myeloid Leukemia: Long-Term Patient Care and Management. J Adv Pract Oncol. 2016;7(1):42-54

Bettiol A, Marconi E, Lombardi N, Crescioli G, Gherlinzoni F, Walley T, et al. Pattern of Use and Long-Term Safety of Tyrosine Kinase Inhibitors: A Decade of Real-World Management of Chronic Myeloid Leukemia. Clin Drug Investig. 2018;38(9):837-44

Brasil. Ministério da Saúde. Fundação Oswaldo Cruz. Agência Nacional de Vigilância Sanitária. Documento de referência para o Programa Nacional de Segurança do Paciente. Brasília: Ministério da Saúde; 2014a.

Brasil. Ministério da Saúde. Protocolo Clínico e Diretrizes Terapêuticas da Leucemia Mieloide Crônica do Adulto. 2020. Available from: http://conitec.gov.br/images/Consultas/Relatorios/2020/PCDT_ LeucemiaMieloideCronicaAdulto_CP_02_2020.pdf. Accessed on: May 20, 2021.

Brasil. Ministério da Saúde. Reação em cadeia da polimerase - transcriptase reversa (RT-PCR) qualitativa e quantitativa (RT-PCR) e Hibridização in situ (ISH) para o diagnóstico e monitoramento da Leucemia Mieloide Crônica (LMC) e da Leucemia Linfoblástica Aguda cromossoma Philadelphia positivo (LLA Ph+). 2019. Available from: http://conitec.gov. br/images/Consultas/Relatorios/2019/RELATORIO_RTPCR_ISH_RTqPCR_ Leucemias_Ph_positivo_CP_38_2019.pdf. Accessed on: May 20, 2021. 
Brasil. Ministério da Saúde. Secretaria de Ciência, Tecnologia e Insumos Estratégicos. Departamento de Ciência e Tecnologia. Diretrizes metodológicas: análise de impacto orçamentário: manual para o Sistema de Saúde do Brasil/Ministério da Saúde, Secretaria de Ciência, Tecnologia e Insumos Estratégicos. Departamento de Ciência e Tecnologia. 1ª ed. Brasília: Ministério da Saúde; 2014b.

Breccia M, Colafigli G, Molica M, Alimena G. Adverse events associated with tyrosine kinase inhibitors for the treatment of chronic myeloid leukemia. Expert Opin Drug Saf. 2016;15(4):525-33.

Caldemeyer L, Dugan M, Edwards J, Akard L. Long-Term Side Effects of Tyrosine Kinase Inhibitors in Chronic Myeloid Leukemia. Curr Hematol Malig Rep. 2016;11(2):71-9.

Cortes JE, Saglio G, Kantarjian HM, Baccarani M, Mayer J, Boqué C, et al. Final 5-Year Study Results of DASISION: The Dasatinib Versus Imatinib Study in Treatment-Naïve Chronic Myeloid Leukemia Patients Trial. J Clin Oncol. 2016;34(20):2333-40.

Costa RS da, Machado Fernandes M, Soler O, Bahia L. Estratégias políticas que norteiam a incorporação de tecnologias: Avaliação de Tecnologias em Saúde em oncologia. JBES. 2017;9(1):30-8.

Cross NCP, White HE, Colomer D, Ehrencrona H, Foroni L, Gottardi E, et al. Laboratory recommendations for scoring deep molecular responses following treatment for chronic myeloid leukemia. Leukemia. 2015;29(5):999-1003.

Druker BJ, Guilhot F, O'Brien SG, Gathmann I, Kantarjian H, Gattermann N, et al. Five-Year Follow-up of Patients Receiving Imatinib for Chronic Myeloid Leukemia. N Engl J Med. 2006;355(23):2408-17.

Elias F, Gebran A, Said C, Beker RV, Ammar W. Budget Impact of TreatmentFree Remission in Treating Chronic-Phase Philadelphia-Positive Chronic Myeloid Leukemia in Lebanon. J Glob Oncol. 2019;5:1-7.

Etienne G, Guilhot J, Rea D, Rigal-Huguet F, Nicolini F, Charbonnier A, et al. Long-Term Follow-Up of the French Stop Imatinib (STIM1) Study in Patients With Chronic Myeloid Leukemia. J Clin Oncol. 2016;35(3):298-305.

Fachi MM, Tonin FS, Leonart LP, Aguiar KS, Lenzi L, Figueiredo BC, et al. Comparative efficacy and safety of tyrosine kinase inhibitors for chronic myeloid leukaemia: A systematic review and network meta-analysis. Eur J Cancer. 2018;104:9-20.

Ferreira-Da-Silva AL, Ribeiro RA, Santos VCC, Elias FTS, d'Oliveira ALP, Polanczyk CA. Diretriz para análises de impacto orçamentário de tecnologias em saúde no Brasil. Cad Saúde Pública. 2012;28(7):1223-38.

Goulart VP. Avaliação do uso de inibidores de tirosina quinase em pacientes com leucemia mielóide crônica. [dissertação de mestrado em Programa de Pós-Graduação em Ciências Farmacêuticas]. Universidade Federal de Santa Maria; 2016. Available from: http://repositorio.ufsm.br/ handle/1/18069. Access on: Jan. 20, 2021.

Hehlmann R, Cortes JE, Zyczynski T, Gambacorti-Passerini C, Goldberg SL, Mauro MJ, et al. Tyrosine kinase inhibitor interruptions, discontinuations and switching in patients with chronic-phase chronic myeloid leukemia in routine clinical practice: SIMPLICITY. Am J Hematol. 2019;94(1):46-54.

Hehlmann R, Hochhaus A, Baccarani M; European LeukemiaNet. Chronic myeloid leukaemia. Lancet. 2007;370(9584):342-50.

Hehlmann R, Müller MC, Lauseker M, Hanfstein B, Fabarius A, Schreiber A, et al. Deep molecular response is reached by the majority of patients treated with imatinib, predicts survival, and is achieved more quickly by optimized high-dose imatinib: results from the randomized CML-study IV. J Clin Oncol. 2014;32(5):415-23.

Heisterkamp N, Stephenson JR, Groffen J, Hansen PF, de Klein A, Bartram CR, et al. Localization of the c-ab1 oncogene adjacent to a translocation break point in chronic myelocytic leukaemia. Nature. 1983:306(5940):239-42.
Hochhaus A, O'Brien SG, Guilhot F, Druker BJ, Branford S, Foroni L, et al. Sixyear follow-up of patients receiving imatinib for the first-line treatment of chronic myeloid leukemia. Leukemia. 2009;23(6):1054-61.

Huang $X$, Cortes J, Kantarjian H. Estimations of the increasing prevalence and plateau prevalence of chronic myeloid leukemia in the era of tyrosine kinase inhibitor therapy. Cancer. 2012;118(12):3123-7.

Hughes T, Deininger M, Hochhaus A, Branford S, Radich J, Kaeda J, et al. Monitoring CML patients responding to treatment with tyrosine kinase inhibitors: review and recommendations for harmonizing current methodology for detecting BCR-ABL transcripts and kinase domain mutations and for expressing results. Blood. 2006;108(1):28-37.

Inca - Instituto Nacional de Câncer José Alencar Gomes da Silva. Ministério da Saúde. Estimativa 2020 Incidência de Câncer no Brasil. 2019. Available from: http://www.inca.gov.br. Accessed on: May 20, 2021.

Kantarjian H, O'Brien S, Jabbour E, Garcia-Manero G, Quintas-Cardama A, Shan J, et al. Improved survival in chronic myeloid leukemia since the introduction of imatinib therapy: a single-institution historical experience. Blood. 2012;119(9):1981-7.

Kizaki M, Takahashi N, Iriyama N, Okamoto S, Ono T, Usui N, et al. Efficacy and safety of tyrosine kinase inhibitors for newly diagnosed chronicphase chronic myeloid leukemia over a 5-year period: results from the Japanese registry obtained by the New TARGET system. Int J Hematol. 2019;109(4):426-39.

Marin D, Bazeos A, Mahon FX, Eliasson L, Milojkovic D, Bua M, et al. Adherence is the critical factor for achieving molecular responses in patients with chronic myeloid leukemia who achieve complete cytogenetic responses on imatinib. J Clin Oncol. 2010;28(14):2381-8.

Menon $\mathrm{H}$. Non-adherence to CML therapy and its clinical implications in India. Natl Med J India. 2017;30(3):142-7.

Noens L, van Lierde MA, De Bock R, Verhoef G, Zachée P, Berneman Z, et al. Prevalence, determinants, and outcomes of nonadherence to imatinib therapy in patients with chronic myeloid leukemia: the ADAGIO study. Blood. 2009;113(22):5401-11.

O'Brien SG, Guilhot F, Larson RA, Gathmann I, Baccarani M, Cervantes F, et al. Imatinib compared with interferon and low-dose cytarabine for newly diagnosed chronic-phase chronic myeloid leukemia. N Engl J Med. 2003;348(11):994-1004.

Rea D. Management of adverse events associated with tyrosine kinase inhibitors in chronic myeloid leukemia. Ann Hematol. 2015;94 Suppl 2:S149-58.

Saußele S, Richter J, Hochhaus A, Mahon FX. The concept of treatment-free remission in chronic myeloid leukemia. Leukemia. 2016;30(8):1638-47.

Saussele S, Richter J, Guilhot J, Gruber FX, Hjorth-Hansen H, Almeida A, et al. Discontinuation of tyrosine kinase inhibitor therapy in chronic myeloid leukaemia (EURO-SKI): a prespecified interim analysis of a prospective, multicentre, non-randomised, trial. Lancet Oncol. 2018;19(6):747-57.

Shih YT, Cortes JE, Kantarjian HM. Treatment Value of Second-generation BcrAbl1 TKIs Compared With Imatinib to Achieve Treatment-free Remission in Patients With Chronic Myeloid Leukemia: a Modelling Study. Lancet Haematol. 2019;6(8):e398-e408.

Takahashi N, Kyo T, Maeda Y, Sugihara T, Usuki K, Kawaguchi T, et al. Discontinuation of imatinib in Japanese patients with chronic myeloid leukemia. Haematologica. 2012;97(6):903-6.

Thielen N, van der Holt B, Cornelissen JJ, Verhoef GE, Gussinklo T, Biemond $B J$, et al. Imatinib discontinuation in chronic phase myeloid leukaemia patients in sustained complete molecular response: a randomised trial of the Dutch-Belgian Cooperative Trial for Haemato-Oncology (HOVON). Eur J Cancer. 2013;49(15):3242-6.

Yamazaki K, Inagaki N, Moldaver D, Viana R, Kimura S. Budget impact analysis of treatment-free remission in nilotinib-treated Japanese chronic myeloid leukemia patients. Cancer Sci. 2020;111(7):2526-35. 